\title{
Entre les moutons et les antivaxs : réactions des médias sociaux aux nouvelles sur les vaccins contre la COVID-19 publiées par les agences de presse canadiennes, et recommandations pour contrer l'hésitation à l'égard de la vaccination
}

\author{
Lisa Tang ${ }^{1 \star}$, Sabrina Douglas ${ }^{1}$, Amar Laila ${ }^{1}$
}

\section{Résumé}

Contexte : Pour créer une initiative de santé publique réussie qui permet de contrer l'hésitation à l'égard de la vaccination et de promouvoir l'acceptation de la vaccination, il est essentiel d'acquérir une solide compréhension des croyances, des attitudes et des perceptions subjectives de la population à l'égard du risque.

Méthodes : Une analyse qualitative du discours sur les vaccins contre la maladie à coronavirus 2019 (COVID-19) provenant de 3731 publications dans les médias sociaux sur les comptes Twitter et Facebook de six agences de presse canadiennes a été utilisée pour déterminer les perceptions, les attitudes, les croyances et les intentions des commentateurs dans les médias sociaux des agences de presse canadiennes à l'égard des vaccins contre la COVID-19.

Résultats : Quatre principaux thèmes ont été dégagés : 1) préoccupations relatives à l'innocuité et à l'efficacité des vaccins contre la COVID-19; 2) théories de conspiration découlant de la méfiance à l'égard du gouvernement et d'autres organisations; 3) un vaccin contre la COVID-19 est inutile, parce que le virus n'est pas dangereux; et 4) la confiance dans les vaccins contre la COVID-19 comme solution sécuritaire. En se fondant sur les thèmes et les sous-thèmes, plusieurs recommandations clés en matière de communication ont été élaborées pour promouvoir l'acceptation des vaccins contre la COVID-19, notamment des infographies parrainées par la Santé publique qui mettent en évidence les avantages des vaccins pour ceux qui l'ont reçu, l'éducation du public au sujet du contenu et de I'innocuité des vaccins et l'établissement d'un lien émotionnel grâce aux histoires personnelles des personnes touchées par la COVID-19.

Conclusion : Des considérations particulières, comme le fait de tirer parti de la confiance du public dans les professionnels de la santé pour agir comme liaison entre la santé publique et le public canadien afin de communiquer les avantages des vaccins contre la COVID-19 et ses variants pourraient aider à réduire l'hésitation à l'égard des vaccins contre la COVID-19.
Cette oeuvre est mise à la disposition selon les termes de la licence internationale Creative Commons Attribution 4.0

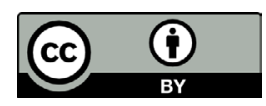

Affiliation

1 Département des relations familiales et de la nutrition appliquée, Université de Guelph, Guelph, ON

\section{*Correspondance :}

lisa.tang@uoguelph.ca

Citation proposée : Tang L, Douglas S, Laila A. Entre les moutons et les antivaxs : réactions des médias sociaux aux nouvelles sur les vaccins contre la COVID-19 publiées par les agences de presse canadiennes, et recommandations pour contrer l'hésitation à l'égard de la vaccination. Relevé des maladies transmissibles au Canada 2021;47(12):582-92. https://doi.org/10.14745/ccdr.v47i12a03f

Mots-clés : hésitation face à la vaccination, médias sociaux, communication sur la santé, COVID-19, vaccins

\section{Introduction}

Le coronavirus du syndrome respiratoire aigu sévère 2

(SRAS-CoV-2) est un agent pathogène respiratoire infectieux responsable de la maladie à coronavirus 2019 (COVID-19) (1). Pour ralentir la propagation de la COVID-19, de nombreuses régions du Canada ont institué l'utilisation de masques à I'intérieur et la distanciation physique. Le 23 mars 2020, le premier ministre Justin Trudeau a exhorté les Canadiens à « rentrer à la maison et à rester chez eux » et à suivre les 
recommandations sur la distanciation physique. À la suite de preuves croissantes de propagation asymptomatique, I'administratrice en chef de la santé publique du Canada a recommandé, le 6 avril 2020, que les Canadiens portent un masque non médical (2). Même avec ces mesures d'atténuation, en septembre 2021, il y avait plus de 27000 décès au Canada, et plus de 4,6 millions de décès dans le monde $(3,4)$. Étant donné que les vaccins constituent l'intervention de santé publique la plus efficace et la plus importante pour prévenir la propagation des maladies infectieuses (5), il est maintenant bien accepté qu'un vaccin contre la COVID-19 est le meilleur moyen de développer une immunité personnelle et collective $(6,7)$. En septembre 2020, le processus accéléré d'approbation des vaccins contre la COVID-19 a été autorisé au Canada (8), ce qui a permis l'approbation des vaccins de Pfizer-BioNTech, Moderna, AstraZeneca et Janssen entre la fin de 2020 et le début de 2021 (9).

La recherche a démontré que la confiance du public à l'égard des vaccins est demeurée faible au cours des dernières années et demeure un problème dynamique et complexe (10-12). Le manque de confiance dans la vaccination a entraîné une hésitation à l'égard de la vaccination, que l'Organisation mondiale de la Santé considère comme l'une des 10 principales menaces à la santé mondiale (13). L'hésitation à se faire vacciner est définie comme un refus ou un retard dans l'acceptation d'un vaccin disponible et est propre au contexte, ce qui signifie qu'une personne peut refuser certains vaccins et en accepter d'autres (14). On a constaté que les technologies de communication numérique, comme les médias sociaux, propagent la désinformation liée à la vaccination (15) qui contribue à I'hésitation à se faire vacciner (16).

Il existe des possibilités de tirer parti de l'utilisation des médias sociaux pour les initiatives de santé publique qui luttent contre la désinformation sur les vaccins et augmentent les taux de vaccination $(16,17)$. Il s'agit d'un facteur important, car l'Enquête sur la santé dans les collectivités canadiennes montre que $75 \%$ des Canadiens de 12 ans et plus seraient quelque peu ou très susceptibles de recevoir les vaccins contre la COVID-19 (18). Les résultats de l'Institut Angus Reid ont révélé que $48 \%$ des Canadiens ont déclaré qu'ils recevraient un vaccin contre la COVID-19 lorsqu'il serait disponible, que $38 \%$ finiraient par le recevoir, mais pas immédiatement, que $14 \%$ ne le recevraient pas et que $7 \%$ étaient incertains (19). Il est important de tenir compte de ces attitudes à l'égard de la vaccination contre la COVID-19 étant donné qu'une grande proportion de la population doit être vaccinée pour atteindre l'immunité collective (20).

Afin de créer des initiatives de santé publique efficaces pour contrer l'hésitation à l'égard de la vaccination et promouvoir l'acceptation de la vaccination, il est nécessaire de comprendre les croyances, les attitudes et les perceptions subjectives de la population à l'égard du risque (21). Une étude récente sur I'intention d'obtenir des vaccins contre la COVID-19 a révélé que les avantages perçus et les obstacles jouaient un rôle dans I'intention de recevoir les vaccins contre la COVID-19 (22). Neubaum et Krämern ont déclaré que les médias sociaux peuvent servir de "fenêtre sur le public », donnant un aperçu de la perception et de l'opinion du public. La recherche a montré que les utilisateurs des médias sociaux peuvent se sentir habilités à dire leurs pensées et leurs opinions lorsqu'ils voient des publications qui correspondent à leurs croyances $(23,24)$ et lorsqu'ils peuvent le faire de façon anonyme (25). Ces commentaires en ligne constituent une source d'information exacte et fiable sur les attitudes et les perceptions du public qui font surface pendant une crise de santé (26). Par exemple, une étude récente a utilisé des messages sur Twitter en anglais pour examiner les perceptions du public à l'égard des mesures de distanciation sociale pour la COVID-19 (27) et a constaté que leurs résultats reflétaient les attitudes et les opinions d'un grand sondage d'opinion publique réalisé aux États-Unis au cours de la même période $(28,29)$. Ensemble, les médias sociaux pourraient servir à mieux comprendre les points de vue de la population canadienne sur les questions de santé publique, y compris les perceptions, les croyances, les attitudes et les intentions à l'égard de la vaccination contre la COVID-19.

Au cours des derniers mois, les perceptions et les attitudes à l'égard de la prise d'un vaccin contre la COVID-19 (30-32) ont été étudiées, et de plus en plus de recherches ont porté sur les perceptions et les attitudes exprimées sur les médias sociaux (33-36). Afin de mieux éclairer les recommandations en matière de santé publique pour contrer la réticence à l'égard de la vaccination au Canada, des recherches plus poussées qui examinent le discours sur les médias sociaux, soit Twitter et Facebook, en réponse aux déclarations sur les vaccins contre la COVID-19 des agences de presse canadiennes pourraient aider à fournir une compréhension plus complète des attitudes, des croyances et des intentions des Canadiens à l'égard des vaccins contre la COVID-19.

\section{Méthodes}

\section{Collecte de données}

Six agences de presse canadiennes populaires ont été choisies, plus précisément Global News, la Société Radio-Canada (SRC), Canadian Television Network (CTV), le Globe and Mail, Maclean's et The National Post afin de cerner les perceptions, les attitudes, les croyances et les intentions des commentateurs dans les médias sociaux des agences de presse canadiennes à l'égard des vaccins contre la COVID-19. II s'agit des principaux fournisseurs de contenu de nouvelles nationales au Canada qui diffusent les nouvelles à la télévision (Global News, SRC, CTV) ou dans la presse écrite (The Globe and Mail, Maclean's, The National Post), ainsi qu'en ligne. Comparée à une analyse quantitative, qui fournit des informations sur les schémas d'hésitation à l'égard de la vaccination parmi les populations, une approche qualitative offre une analyse plus approfondie des aspects socioculturels de l'hésitation à l'égard de la vaccination (37). Par conséquent, 
une approche qualitative a été choisie pour permettre une exploration approfondie des nuances et des complexités de I'hésitation à l'égard de la vaccination chez les Canadiens.

Les publications sur les comptes Twitter et Facebook des six agences de presse canadiennes énumérées ci-dessus ont été surveillées pour savoir quand un article sur les vaccins contre la COVID-19 a été diffusé sur leur compte Twitter et Facebook. Twitter et Facebook ont été choisis parce que les commentaires sur ces plateformes ont été utilisés pour répondre aux questions de recherche sur l'hésitation à l'égard de la vaccination dans des études antérieures $(33,38,39)$ et que les deux plateformes permettent aux agences de presse de donner le lien vers les articles sur leur site Web. Toutes les données ont été recueillies entre juillet et septembre 2020, et seules les publications en anglais ont été recueillies aux fins d'analyse. Chaque publication sur les médias sociaux comportait un lien vers leur article de presse respectif et souvent un commentaire invitant la participation sur la plateforme. Ces agences de presse ont été choisies parce qu'elles sont représentatives à l'échelle nationale et qu'elles utilisent des pratiques de reportage crédibles et des tendances politiques variées. Tous les auteurs de commentaires sont des utilisateurs de médias sociaux ayant des comptes sur Twitter et/ou Facebook. Les auteurs ont cherché des articles de presse qui comprenaient de l'information sur le développement ou l'achat de vaccins contre la COVID-19 ou des rapports sur les résultats du sondage sur la vaccination. Sept jours après que I'article de presse sur la COVID-19 a été diffusé sur le compte de médias sociaux de l'organisation, tous les commentaires affichés ont été recueillis. Un délai de sept jours était suffisant pour recueillir les commentaires des médias sociaux sur cet article, car peu de commentaires ont été affichés après cette période.

Au total, six articles (un article par organisme d'information publié sur Twitter et Facebook) et 4095 commentaires ont été recueillis aux fins d'analyse. Les données ont ensuite été numérisées pour détecter les pourriels, qui étaient définis comme des insultes à l'endroit d'autres auteurs de commentaires, des commentaires qui ne portaient pas sur les vaccins contre la COVID-19, des commentaires qui n'étaient pas lisibles (e.g. utilisation de caractères uniquement) et des images (e.g. GIFS). Au total, 364 messages contenant des pourriels et des commentaires non pertinents ont été supprimés, et les images contenant du texte, si elles étaient liées à la COVID-19, ont été transcrites en mots. Une fois le nettoyage des données terminé, il restait au total 3731 publications à analyser. Le tableau 1 indique le nombre de publications par agence de presse et les liens vers chaque article.

Cette étude de recherche reposait exclusivement sur des données accessibles au public et certaines sources étaient anonymes ou non identifiables; par conséquent, l'approbation éthique n'était pas requise. Cela est conforme à une recherche canadienne semblable utilisant du contenu de médias sociaux accessible au public (33).
Tableau 1 : Nombre total de publications sur Twitter et Facebook combinées pour chaque agence de presse utilisé dans l'analyse et liens vers l'article sur les vaccins contre la COVID-19 de chaque agence de presse publié sur leurs comptes de médias sociaux respectifs

\begin{tabular}{|c|c|c|}
\hline $\begin{array}{l}\text { Agence de } \\
\text { presse }\end{array}$ & $\begin{array}{l}\text { Nombre de } \\
\text { publications }\end{array}$ & Lien vers I'article \\
\hline $\begin{array}{l}\text { National } \\
\text { Post }\end{array}$ & 308 & $\begin{array}{l}\text { https://nationalpost.com/health/ } \\
\text { which-canadians-get-the-covid-19- } \\
\text { vaccine-first-experts-are-struggling- } \\
\text { to-decide }\end{array}$ \\
\hline Maclean's & 642 & $\begin{array}{l}\text { https://www.macleans.ca/society/ } \\
\text { health/how-anti-vaxxers-could-disrupt- } \\
\text { the-cure-for-the-covid-19-pandemic/ }\end{array}$ \\
\hline $\begin{array}{l}\text { The Globe } \\
\text { and Mail }\end{array}$ & 70 & $\begin{array}{l}\text { https://www.theglobeandmail.com/ } \\
\text { canada/article-moderna-inc-says- } \\
\text { its-covid-19-vaccine-shows-positive- } \\
\text { results-among/ }\end{array}$ \\
\hline Global News & 745 & $\begin{array}{l}\text { https://globalnews.ca/news/7251593/ } \\
\text { canada-pfizer-coronavirus-vaccine/ }\end{array}$ \\
\hline SRC & 498 & $\begin{array}{l}\text { https://www.cbc.ca/news/world/ } \\
\text { coronavirus-covid19-world- } \\
\text { sept4-1.5712020 }\end{array}$ \\
\hline CTV & 1468 & $\begin{array}{l}\text { https://www.ctvnews.ca/health/ } \\
\text { coronavirus/feds-sign-deals-with- } \\
\text { novavax-and-johnson-johnson- } \\
\text { to-secure-millions-of-vaccine- } \\
\text { doses-1.5085911 }\end{array}$ \\
\hline
\end{tabular}

Abréviations : COVID-19, maladie à coronavirus 2019; CTV, Canadian Television Network SRC, Société Radio-Canada

\section{Analyse}

Des messages originaux provenant des comptes Twitter et Facebook de chaque agence de presse, ainsi que des commentaires connexes, ont été importés dans NVivo-12 (QSR International, 2019). Utilisant Clarke et Braun (40) comme guide, chacun des chercheurs de cette étude a effectué une analyse thématique pour déterminer les thèmes comme unité d'analyse. L'analyse a nécessité le codage indépendant de chaque commentaire et la réponse sur une période de 10 semaines. Les chercheurs se sont réunis toutes les deux semaines pour examiner les différences entre les codes et en discuter, ce qui est devenu le fondement des thèmes (40). Selon la documentation antérieure sur la réticence à l'égard de la vaccination, les perceptions, les attitudes, les croyances et les intentions ont été utilisées comme concepts de sensibilisation pour aborder l'analyse qualitative. Les concepts de sensibilisation désignent les idées générales qui servent de point de départ pour les chercheurs qui abordent une question de recherche qualitative (41). En utilisant ces concepts de sensibilisation comme guide d'analyse, les auteurs ont ensuite utilisé l'analyse inductive pour permettre aux thèmes et aux tendances de se dégager des données (41). Les trois chercheurs ont remarqué des thèmes semblables parmi les données, et une fois le codage terminé, tous les chercheurs se sont réunis pour finaliser la liste des thèmes et sous-thèmes convenus. 


\section{Résultats}

Quatre thèmes sont ressortis des commentaires recueillis en réponse aux publications des agences de presse sur les médias sociaux. Pour chaque thème, des sous-thèmes ont également été relevés. La plupart des commentaires et des réponses sur les médias sociaux exprimaient des attitudes et des opinions négatives à l'égard des vaccins contre la COVID-19, tandis que certains exprimaient des croyances et des attitudes positives. Chaque thème est décrit dans les pages suivantes, où des citations illustratives ont été utilisées pour mettre les thèmes en contexte. Le tableau 2 présente un résumé des thèmes et des citations supplémentaires.

\section{Tableau 2 : Citations supplémentaires de commentaires sur les médias sociaux en réponse aux publications sur Twitter et Facebook sur les vaccins contre la COVID-19 des agences de presse canadiennes, organisées par thème et sous-thème}

\begin{tabular}{|c|c|c|}
\hline Thème & Sous-thème & Citation [Traduction] \\
\hline \multirow{5}{*}{$\begin{array}{l}\text { Innocuité et } \\
\text { efficacité des } \\
\text { vaccins contre la } \\
\text { COVID-19 }\end{array}$} & $\begin{array}{l}\text { Pressions politiques } \\
\text { influençant la production } \\
\text { de vaccins }\end{array}$ & $\begin{array}{l}\text { "Ces scientifiques subissent d'énormes pressions pour que les gouvernements fassent avancer } \\
\text { les choses et mettent en place un vaccin, et c'est ainsi que nous nous retrouvons avec des } \\
\text { erreurs - CTV, Facebook } \\
\text { "Prendriez-vous un vaccin qui n'a pas fait l'objet d'essais complets? DJT [Donald J Trump] } \\
\text { propose de renoncer aux essais de phase } 3 \text { afin d'accélérer la production d'un vaccin » - SRC, } \\
\text { Twitter }\end{array}$ \\
\hline & $\begin{array}{l}\text { D'autres doivent d'abord } \\
\text { prouver l'innocuité }\end{array}$ & $\begin{array}{l}\text { "J'attendrai que tous les héros passent en premier, s'ils survivent, alors peut-être »- CTV, Twitter } \\
\text { "Non. Pas avant de voir ce qui arrive à tous les zélés. Je ne suis pas un cobaye »-CTV, Twitter } \\
\text { "Les défendeurs peuvent faire la queue pour être des cobayes pendant que les gens intelligents } \\
\text { attendent de voir ce qui se passe. » - Global News, Twitter } \\
\text { "Je pense que tous nos charmants politiciens devraient être les premiers à l'obtenir, et nous } \\
\text { pouvons tous attendre six mois pour voir comment les choses se passeront. » - CTV, Facebook }\end{array}$ \\
\hline & Vaccin fait à la hâte & $\begin{array}{l}\text { "Je ne suis pas un cobaye. J'attendrai de } 5 \text { à } 10 \text { ans qu'une étude à long terme soit examinée par } \\
\text { des pairs et d'être certain que les effets secondaires des vaccins ne sont pas pires que les efforts } \\
\text { qu'il faut déployer pour éviter la COVID-19. »-SRC, Facebook } \\
\text { " Quiconque est assez stupide pour se faire injecter un vaccin fait à la hâte et insuffisamment testé } \\
\text { mérite tous les effets secondaires. » - CTV, Facebook } \\
\text { " Non... Je ne suis pas antivax... Je suis contre le fait d'être un cobaye pour un vaccin fait à la hâte } \\
\text { qui n'a pas été testé correctement » - CTV, Facebook } \\
\text { " Je ne suis pas contre les vaccins, mais je ne recevrai pas celui-là. C'est tout simplement trop } \\
\text { rapide et pas assez testé pour que je veuille prendre ça. " - Maclean's, Facebook } \\
\text { "Vous savez qu'il faut environ } 10 \text { ans pour développer et tester correctement un vaccin? Allez-y et } \\
\text { faites confiance à quelque chose qui a été mis au point en quatre mois et qui n'a aucune donnée } \\
\text { sur l'effet à long terme, mais si vous vous respectez un peu, vous attendrez d'avoir des preuves } \\
\text { irréfutables que ce vaccin est sûr à } 100 \% \text { et qu'il y a PEU de chances que des complications } \\
\text { se produisent, comme tous les autres vaccins rigoureusement testés dont l'innocuité est } \\
\text { prouvée. »- National Post, Facebook }\end{array}$ \\
\hline & $\begin{array}{l}\text { Préoccupation relative } \\
\text { aux ingrédients }\end{array}$ & $\begin{array}{l}\text { «Allez-y et moi aussi, mais ne jugez pas les autres qui n'ont pas envie de mettre des produits } \\
\text { chimiques inconnus dans leur corps »- CTV, Facebook } \\
\text { «... vérifiez ce qu'il y a dans les vaccins et ce qu'ils font vraiment et vérifiez s'ils ne veulent } \\
\text { pas de produits chimiques comme le formaldéhyde, le mercure et l'aluminium dans leur } \\
\text { corps »- Maclean's, Facebook } \\
\text { "Lisez l'encart et voyez ce qu'il contient. ADN fœtal Oui, des cellules fœtales avortées. Les } \\
\text { produits chimiques toxiques sont incompréhensibles. Vous exigez un masque pour notre santé et } \\
\text { vous injectez ensuite ces toxines directement dans notre sang. FAITES DES RECHERCHES sur leur } \\
\text { contenu! »- Global News, Twitter }\end{array}$ \\
\hline & Vaccin contre les variants & $\begin{array}{l}\text { « Nous ne connaissons pas encore, ou du moins on ne nous dit pas le taux de mutation de } \\
\text { la COVID... la vaccination peut être fréquente et peut-être sans effet réel. » - National Post, } \\
\text { Facebook } \\
\text { "La COVID est déjà en train de muter, alors bonne chance avec ça »- SRC, Facebook } \\
\text { «Pensez au nombre de fois où le virus se sera transformé au moment où le vaccin sera } \\
\text { distribué... »- SRC, Facebook }\end{array}$ \\
\hline
\end{tabular}


Tableau 2 : Citations supplémentaires de commentaires sur les médias sociaux en réponse aux publications sur Twitter et Facebook sur les vaccins contre la COVID-19 des agences de presse canadiennes, organisées par thème et sous-thème (suite)

\begin{tabular}{|c|c|c|}
\hline Thème & Sous-thème & Citation [Traduction] \\
\hline \multirow{3}{*}{$\begin{array}{l}\text { Théories du } \\
\text { complot découlant } \\
\text { de la méfiance } \\
\text { à l'égard du } \\
\text { gouvernement } \\
\text { et d'autres } \\
\text { organisations }\end{array}$} & $\begin{array}{l}\text { Méfiance générale } \\
\text { à l'égard du } \\
\text { gouvernement }\end{array}$ & $\begin{array}{l}\text { «Je ne fais plus confiance à notre gouvernement et je ne servirai pas de cobaye. »- Global News, } \\
\text { Twitter } \\
\text { "Qui veut se faire injecter quelque chose dans le bras d'un gouvernement qui a fait l'objet de } \\
3 \text { enquêtes sur l'éthique, qui est très loin de tout ce qui ressemble à la "transparence" qu'il devrait } \\
\text { être traité comme criminel. JT [Justin Trudeau] - Monsieur mieux-que-tout-le-monde est sur notre } \\
\text { dos depuis assez longtemps, pas besoin de m'injecter dans le bras. De toute évidence, on ne doit } \\
\text { PAS vous faire confiance. » - CTV, Facebook } \\
\text { "... Ce n'est pas parce que le gouvernement dit que c'est correct et qu'il va de l'avant avec la } \\
\text { création et les essais que j'ai confiance en lui. » - Global News, Facebook } \\
\text { « } 0 \text { \% de confiance dans le système de santé canadien pour fournir une version sécuritaire de la } \\
\text { vaccination contre la COVID-19. »- Global News, Twitter } \\
\text { "Les scientifiques peuvent être achetés comme les politiciens. Cessez de penser naïvement que le } \\
\text { gouvernement veut ce qu'il y a de mieux pour nous. »-CTV, Facebook }\end{array}$ \\
\hline & $\begin{array}{l}\text { Le vaccin contre la } \\
\text { COVID-19 modifiera } \\
\text { votre ADN }\end{array}$ & $\begin{array}{l}\text { "Vous rendez-vous compte que le nouveau vaccin d'ARNm que BigPharma vante comme le } \\
\text { sauveur de la COVID-19 modifie en fait votre ADN? Pas étonnant qu'ils aient mis Gates au premier } \\
\text { plan pour le vendre. Ils l'appellent "Le logiciel de la vie" ». - Global News, Twitter } \\
\text { "Allez donc modifier votre ADN pour le reste de votre vie et celle de vos enfants. »- Global } \\
\text { News, Twitter } \\
\text { "Pourquoi est-ce que je le prendrais sachant que I'ADN est modifié? Cela signifie changer vos } \\
\text { génomes et votre ADN... Vous devriez vous demander pourquoi ils se précipitent pour l'injecter à } \\
\text { la population. »- CTV, Facebook }\end{array}$ \\
\hline & $\begin{array}{l}\text { Micropuces et } \\
\text { nanotechnologie }\end{array}$ & $\begin{array}{l}\text { «Je ne veux pas qu'on m'injecte une micropuce de Bill Gates, c'est un dispositif de contrôle de } \\
\text { l'esprit qui peut simplement vous mettre sur une autre planète et vous censurer. " - Global News, } \\
\text { Twitter } \\
\text { "Ceux d'entre nous qui ont un système immunitaire fort survivront très bien sans le vaccin de } \\
\text { l'enfer de Gates qui, a-t-il admis, tuera plus de } 700000 \text { personnes. Allez donc vous faire mettre } \\
\text { une micropuce comme les vaches. » - Maclean's, Facebook } \\
\text { « Il y a une énorme différence entre une puce dans un téléphone ou un appareil électronique et } \\
\text { une puce dans votre corps! Au moins, vous pouvez lasser votre téléphone à la maison. »- CTV, } \\
\text { Facebook }\end{array}$ \\
\hline \multirow{3}{*}{$\begin{array}{l}\text { Le vaccin contre } \\
\text { la COVID-19 est } \\
\text { inutile, parce que } \\
\text { le virus n'est pas } \\
\text { dangereux }\end{array}$} & $\begin{array}{l}\text { C'est simplement une } \\
\text { " campagne de peur » }\end{array}$ & $\begin{array}{l}\text { "Je suis certain que nous pourrions revenir à la vie prépandémie si les médias cessaient tout } \\
\text { simplement de semer la peur " - CTV, Facebook } \\
\text { "Votre propagande de peur, vos décrets sur l'obligation des masques, vos statistiques gonflées } \\
\text { sont tous du n'importe quoi. »-CTV, Facebook } \\
\text { «Encore une fois, notre gouvernement ridicule tient des propos alarmistes. »-CTV, Facebook }\end{array}$ \\
\hline & $\begin{array}{l}\text { La COVID-19 n'est pas } \\
\text { si grave }\end{array}$ & $\begin{array}{l}\text { "Vous aviez de meilleures chances de mourir d'un cancer ou d'un accident de la route ou de toute } \\
\text { autre raison de santé chaque jour que d'être infecté par la COVID-19 ou d'en mourir. "- CTV, } \\
\text { Facebook } \\
\text { "Un vaccin est-il vraiment nécessaire pour une maladie si mortelle qu'il faut la tester pour savoir si } \\
\text { on l'a? - CTV, Facebook } \\
\text { "Ma femme et moi l'avons tous les deux eu (nous sommes tous les deux immunodéficients). } \\
\text { Aucun séjour à l'hôpital, la toux n'a duré qu'environ trois semaines et nous n'avons aucun effet à } \\
\text { long terme. " - CTV, Facebook } \\
\text { "Un vaccin contre un virus avec un taux de mortalité de } 0,03 \% \text { ? Je vais passer, merci! »- The } \\
\text { Globe and Mail, Facebook }\end{array}$ \\
\hline & $\begin{array}{l}\text { Un système immunitaire } \\
\text { solide et un mode de } \\
\text { vie sain suffisent pour } \\
\text { vaincre la COVID-19 }\end{array}$ & $\begin{array}{l}\text { "Je ne suis pas immunodéprimé, je ne suis pas une personne âgée, je suis en bonne santé, et } \\
\text { chaque fois que j'ai eu une grippe, mes propres systèmes de défense l'ont surmontée au moment } \\
\text { normal prévu de l'infection. "- Global News, Facebook } \\
\text { «Mangez, dormez et faites de l'exercice et tout ira bien. Si tout le monde faisait ça, } 80 \text { \% des } \\
\text { problèmes du système de santé seraient évités. " - Global News, Facebook } \\
\text { « J'aimerais être immunisé contre la maladie avec mes propres anticorps naturels. » - Global News, } \\
\text { Facebook } \\
\text { "Manger sainement : aliments non transformés, non génétiquement modifiés, aliments } \\
\text { biologiques; faire de l'exercice; bien dormir; prendre des vitamines; s'exposer au soleil; et la } \\
\text { liste de ce que vous pouvez faire pour rester en santé est longue. Je n'ai pas besoin de produits } \\
\text { chimiques pour rester en santé. Laissez le corps faire son travail et si j'attrape un rhume, une } \\
\text { grippe ou la COVID, je m'en occuperai. "- Global News, Facebook }\end{array}$ \\
\hline
\end{tabular}


Tableau 2 : Citations supplémentaires de commentaires sur les médias sociaux en réponse aux publications sur Twitter et Facebook sur les vaccins contre la COVID-19 des agences de presse canadiennes, organisées par thème et sous-thème (suite)

\begin{tabular}{|c|c|c|}
\hline Thème & Sous-thème & Citation [Traduction] \\
\hline \multirow{3}{*}{$\begin{array}{l}\text { Confiance dans les } \\
\text { vaccins contre la } \\
\text { COVID-19 comme } \\
\text { solution sécuritaire }\end{array}$} & $\begin{array}{l}\text { Confiance envers les } \\
\text { professionnels de } \\
\text { la science et de la } \\
\text { médecine }\end{array}$ & $\begin{array}{l}\text { "J'ai vu les ingrédients et, contrairement à certaines personnes, je ne les interprète pas mal. } \\
\text { Certains ingrédients peuvent sembler louche à quiconque ne comprend pas la chimie. » - Global } \\
\text { News, Twitter } \\
\text { "Je pense que faire des essais sur plus de } 50000 \text { personnes suffit » - Global News, Facebook } \\
\text { "Personne ne distribuera de vaccin non testé. Il ne sera peut-être pas possible de tester la } \\
\text { protection à long terme, mais il sera certainement testé pour sa sécurité et son efficacité. »- CTV, } \\
\text { Facebook } \\
\text { "Comme je l'ai dit, mon plan de gestion des risques consiste à écouter mon médecin de famille } \\
\text { et mon épouse, qui est une infirmière spécialisée en prévention des infections à la retraite. Ces } \\
\text { deux femmes ne m'ont jamais induit en erreur. Je vous souhaite bonne chance avec votre plan de } \\
\text { rechange. » - Maclean's, Facebook } \\
\text { "La raison pour laquelle il peut être créé si rapidement est qu'il s'agit d'un virus que nous } \\
\text { connaissons bien. De plus, je ne sais pas si vous en êtes conscient, mais la recherche et la } \\
\text { technologie ont progressé. »- SRC, Facebook }\end{array}$ \\
\hline & $\begin{array}{l}\text { Préoccupations au sujet } \\
\text { des effets à long terme } \\
\text { de la COVID-19 }\end{array}$ & $\begin{array}{l}\text { "Le problème n'est pas seulement la mortalité liée à la COVID, c'est aussi la gravité de la maladie } \\
\text { et ses effets à long terme. Mais pour l'instant, vous ne mourrez peut-être pas de la COVID, mais } \\
\text { vous pourriez mourir en attendant des soins dans un hôpital surpeuplé rempli de patients infectés } \\
\text { par la COVID. » - Globe and Mail, Facebook } \\
\text { "Presque toutes les personnes interrogées dans les médias, jeunes et moins jeunes, qui l'ont eu } \\
\text { disent qu'elles ne se sentent toujours pas complètement rétablies... certaines ont des pertes de } \\
\text { mémoire, une perte d'énergie, etc. " - CTV, Facebook } \\
\text { "Des personnes en bonne santé peuvent quand même subir des dommages permanents et } \\
\text { mourir »- Global News, Twitter }\end{array}$ \\
\hline & $\begin{array}{l}\text { Intention d'obtenir } \\
\text { les vaccins contre la } \\
\text { COVID-19 pour protéger } \\
\text { les autres et revenir à } \\
\text { une vie «normale" }\end{array}$ & $\begin{array}{l}\text { "Merci aux rares voix de la raison dans une foule de hurlements d'antivaxs. En tant que } \\
\text { personne ayant des proches qui ont des problèmes de santé, je serai le premier à recevoir mon } \\
\text { vaccin. "- CTV, Facebook } \\
\text { "Peut-être que si tout le monde était vacciné, portait un masque et respectait la distance sociale, } \\
\text { la vie reviendrait à la normale } 10 \text { fois plus vite que prévu. »- CTV, Facebook }\end{array}$ \\
\hline
\end{tabular}

Abréviations : COVID-19, maladie à coronavirus 2019; CTV, Canadian Television Network; SRC, Société Radio-Canada

\section{Thème $n^{\circ} 1:$ Innocuité et efficacité des vaccins contre la COVID-19}

Le thème $n^{\circ} 1$ a fait état de préoccupations au sujet des facteurs perçus qui peuvent influer sur l'innocuité et l'efficacité des vaccins, notamment les pressions politiques, la vitesse de développement et les essais, les ingrédients et les échappements immunitaires potentiels de certains variants.

Pressions politiques influençant la production de vaccins : Des préoccupations ont été exprimées au sujet de l'influence perçue des pressions politiques qui précipitent la production de vaccins. Par exemple, un commentateur a dit : «Est-ce que j'obtiendrais le vaccin russe ou le vaccin de Trump pour gagner une élection [?] Aucune chance. " - CTV, Twitter. Un autre commentateur, qui parlait de l'influence des politiciens, a écrit : "Les discours des experts médicaux sont dictés par les politiciens. Faites-leur confiance à vos risques et périls »- Globe and Mail, Facebook.

D'autres doivent d'abord prouver l'innocuité : Une préoccupation commune concernait l'innocuité des vaccins et la croyance qu'il n'y avait pas assez d'essais. De nombreux commentateurs ont fait remarquer que les politiciens devraient recevoir le vaccin en premier pour prouver son innocuité : « Je veux que la Chambre des communes, le Sénat, la gouverneure générale et le premier ministre reçoivent un vaccin spécial! Ensuite, nous attendons un mois pour voir ce qui va se passer! » - CTV, Facebook. Un autre a écrit : « Je vais laisser la masse être le groupe de contrôle et voir ce qui va se passer. C'est peut-être bon, peut-être pas. Le temps et les essais nous le diront. »-SRC, Facebook.

Vaccin fait à la hâte : De nombreux répondants se sont dits préoccupés par le court délai pour le développement des vaccins contre la COVID-19. Un commentateur qui s'est dit ne pas être un " antivax », un mot qui décrit quelqu'un qui s'oppose aux vaccins, a fait remarquer ce qui suit : "Il y aura beaucoup de gens comme moi qui ne sont pas des antivaxs, mais qui le refuseront jusqu'à ce qu'il y ait un délai raisonnable pour les essais et les données appropriés. » - National Post, Facebook.

Préoccupation relative aux ingrédients : Préoccupations relatives à l'innocuité des ingrédients utilisés pour mettre au point les vaccins contre la COVID-19. " Vas-y et mets du tissu fœtal, du mercure et du formaldéhyde dans tes veines, et rentres 
chez toi voir à quel point tu te sens bien! »- Global News, Facebook.

Vaccin contre les variants : Les commentateurs s'inquiétaient de l'efficacité des vaccins après la mutation du virus causant la COVID-19. L'un d'entre eux a écrit ceci : "II y a une réelle possibilité que le virus causant la COVID mute et rende tout vaccin inutile »-Maclean's, Facebook, tandis qu'un autre a dit : «Pensez au nombre de fois où le virus se sera transformé au moment où le vaccin sera distribué... ». - SRC, Facebook.

\section{Thème $\mathrm{n}^{\circ} 2$ : Théories du complot découlant de la méfiance à l'égard du gouvernement et d'autres organisations}

Le thème $\mathrm{n}^{\circ} 2$ portait sur les théories du complot, y compris les micropuces et les changements à l'ADN, exprimées sur les médias sociaux, et fondées sur une méfiance générale à l'égard du gouvernement et des organisations participant à la mise au point des vaccins contre la COVID-19.

Méfiance générale à l'égard du gouvernement : Les articles publiés par les six agences de presse sur les médias sociaux contenaient des commentaires faisant état d'une méfiance à l'égard des gouvernements étrangers et nationaux et des organismes de santé. Un intervenant qui parlait du gouvernement a écrit : "Pas une graine de confiance. Je ne suis pas un cobaye pour les tests de vaccins gouvernementaux »Global News, Twitter.

Le vaccin contre la COVID-19 modifiera I'ADN : Les commentaires au sujet du vaccin modifiant l'ADN étaient courants sur toutes les plateformes des médias sociaux des agences de presse : "C'est fou! Ces personnes ont-elles une idée de ce que ce vaccin implique ?! II va littéralement altérer votre ADN. Pour toujours. »- Global News, Facebook. Un autre commentateur a écrit : "Je n'en ai pas besoin et je ne veux pas que quiconque modifie mon ADN »-Global, News, Twitter.

Micropuces et nanotechnologie : Le discours axé sur les micropuces et la nanotechnologie était courant. Un commentateur a écrit : "Je ne veux pas me faire injecter une micropuce »- CTV, Facebook, tandis qu'un autre a répondu : "Bill Gates peut garder son jus de nanobot, lol. »- Global News, Facebook.

\section{Thème $n^{\circ} 3$ : Le vaccin contre la COVID-19 est inutile, parce que le virus $n$ 'est pas dangereux}

Le thème $\mathrm{n}^{\circ} 3$ a permis de saisir le niveau de préoccupation liée à la gravité perçue de l'infection par la COVID-19 exprimée dans les médias sociaux. Les commentateurs étaient d'avis que la sévérité était surévaluée et qu'un système immunitaire sain était suffisait pour vaincre le virus.
C'est simplement une " campagne de peur ": De nombreux commentateurs étaient d'avis que le virus n'était pas aussi grave que ce que les médias rapportaient. En réponse à une question posée par une agence de presse qui demandait si les gens allaient recevoir le vaccin, un intervenant a répondu : "Encore une fois, notre gouvernement ridicule tient des propos alarmistes. » - CTV, Facebook.

La COVID-19 n'est pas si grave : De nombreux répondants ont souligné qu'un vaccin contre la COVID-19 n'était pas nécessaire parce que le virus n'était pas dangereux. Par exemple, « II a déjà frappé dans ma maison, ma femme et moi sommes à très haut risque, et aucun hôpital pour nous deux, et pourtant nous sommes là! » - CTV, Facebook, alors qu'un autre commentateur a écrit : "Je suis plus susceptible de mourir en descendant mes escaliers que de mourir de la COVID. »- Global News, Twitter.

Un système immunitaire solide et un mode de vie sain suffisent pour vaincre la COVID-19 : Les commentateurs ont expliqué comment être en bonne santé était suffisant pour vaincre le virus : "C'est peut-être la façon dont le monde élimine les faibles. La plupart ont des problèmes sous-jacents et nous sommes en parfaite santé, alors la COVID ne nous préoccupe pas. "- SRC, Facebook. Un autre commentateur a écrit : «Mangez sainement et prenez des vitamines, c'est le meilleur vaccin que nous puissions obtenir ». - CTV, Facebook.

\section{Thème $n^{\circ} 4$ : Confiance dans les vaccins contre la COVID-19 comme solution sécuritaire}

Une minorité de répondants ont exprimé leur confiance dans les vaccins contre la COVID-19 pour prévenir l'infection. Les personnes qui ont confiance dans le vaccin ont exprimé leur confiance dans la science et leur professionnel de la santé, ont exprimé des préoccupations au sujet des effets potentiels à long terme de la COVID-19 et ont estimé que le vaccin était nécessaire pour revenir à la normale.

Confiance envers les professionnels de la science et de la médecine : Les commentateurs ont exprimé leur confiance dans la science derrière les vaccins : "Si Santé Canada approuve un vaccin, je serai dans la première ligne disponible » - CTV, Twitter. Un autre commentateur a écrit : «Inscrivez-moi, étonnamment, je fais confiance à la science et aux mesures de protection médicales en place. Je sais, on n'entend jamais ça. » - National Post, Facebook.

D'autres répondants ont mentionné leur confiance envers les professionnels de la santé : "Si mon Dr le recommande, j'accepte de le prendre. " - Global News, Twitter. Un autre a écrit : "Je suivrai les conseils de mon médecin, car je n'ai pas de rate. »-Global News, Twitter. 
Préoccupations au sujet des effets à long terme de

la COVID-19 : Plusieurs intervenants ont soulevé des préoccupations au sujet des effets potentiels à long terme de I'infection par le virus causant la COVID-19. Un commentateur a écrit : "Le problème, ce n'est pas seulement ceux qui sont morts, mais ceux qui ont survécu, ce qu'ils ont vécu et les effets à plus long terme... »- CTV, Facebook. Un autre a écrit ceci : " Je suis très disposé à le prendre, les effets à long terme de la COVID sont ce qui m'a convaincu »-CTV, Facebook.

Intention d'obtenir les vaccins contre la COVID-19 pour protéger les autres et revenir à une vie " normale ": Les commentateurs ont exprimé l'intention d'obtenir les vaccins contre la COVID-19 pour qu'ils puissent retourner à une vie normale : " Je serai le premier en ligne pour que nous puissions revenir à la normale »-CTV, Facebook. Un autre a écrit : «Dès qu'il sera disponible! Je vais définitivement faire ma part pour protéger les personnes vulnérables »- Global News, Facebook.

En revanche, ceux qui ont exprimé leur intention de recevoir les vaccins contre la COVID-19 ont été ridiculisés. Des commentaires comme celui-ci étaient courants : «Ouais... tous les moutons effrayés feront la queue consciencieusement et ils vont humilier quiconque résiste »-SRC, Twitter.

\section{Discussion}

Cette étude visait à examiner le discours sur les médias sociaux dans les comptes des agences de presse canadiennes en réponse aux articles publiés sur les vaccins contre la COVID-19. Les commentaires sur les articles publiés ont été analysés afin de déterminer les perceptions, les attitudes, les croyances et les intentions à l'égard de la vaccination contre la COVID-19. Notre analyse a dégagé quatre thèmes et un certain nombre de sousthèmes.

Les commentaires exprimant des préoccupations au sujet de I'innocuité et de l'efficacité d'un vaccin contre la COVID-19 étaient courants. Cela concorde avec les recherches antérieures qui ont examiné les raisons de l'hésitation à se faire vacciner, les préoccupations relatives à l'innocuité et à l'efficacité étant le principal facteur d'hésitation à se faire vacciner $(42,43)$. La préoccupation commune au sujet d'un vaccin fait " à la hâte » n'est pas propre à la COVID-19. Les recherches sur les réponses au vaccin contre la grippe H1N1 ont révélé que les gens étaient préoccupés par la mise au point précipitée des vaccins (44). Ces constatations concordent avec notre analyse et sont troublantes, car la recherche a révélé que l'acceptation des vaccins contre la COVID-19 est fortement liée à la perception de l'innocuité (45).

Les auteurs des commentaires étaient préoccupés par les ingrédients dans les vaccins contre la COVID-19. Ces résultats concordent avec les recherches antérieures de Björkman et Sanner (46) qui ont examiné les expériences et les croyances liées à la prise des vaccins contre la grippe H1N1 en Suède.
Cette étude a permis de déterminer que les participants étaient préoccupés par la présence de " substances inconnues » contenues dans le vaccin qui vont dans leur corps (46). Dans l'ensemble, il semble que le manque de compréhension du contenu des vaccins ait constitué un obstacle constant à l'adoption des vaccins.

Les commentateurs sur les médias sociaux étaient préoccupés par les mutations virales de la COVID-19 qui rendent les vaccins inefficaces contre le virus. La recherche a montré que la rapidité de la vaccination peut compenser les dommages causés par des variants plus facilement transmissibles (47). Par conséquent, des messages de santé publique qui répondent aux préoccupations au sujet des variants du virus causant la COVID-19 et qui encouragent l'adoption des vaccins sont nécessaires.

Notre analyse a révélé que l'une des raisons pour lesquelles on appuyait un vaccin contre la COVID-19 était la crainte des effets potentiels à long terme du virus. Cela concorde avec des recherches antérieures qui ont révélé que les perceptions de la gravité de la maladie étaient associées à la volonté de recevoir un vaccin contre la COVID-19 $(43,48,49)$. Une suggestion pour accroître l'adoption des vaccins pourrait être la communication de données locales au moyen d'infographies claires pour illustrer le succès des vaccins contre la COVID-19 pour les personnes qui ont été vaccinées. Cela peut avoir une influence positive sur ceux qui hésitent pour des raisons d'efficacité, l'accent étant mis sur le risque de développer des symptômes à long terme après une infection par la COVID-19. De plus, il ressort clairement de nos résultats et des recherches antérieures $(43,48,50)$ que les fournisseurs de soins de santé sont des participants efficaces à la communication sur les vaccins, car plusieurs commentateurs ont mentionné qu'ils recevraient le vaccin si leur médecin le recommandait.

\section{Limites}

Il faut tenir compte des limites de l'étude dans l'interprétation des résultats. Premièrement, il est probable que les lecteurs qui font des commentaires sur les publications liées au vaccin ont des sentiments très négatifs à l'égard des vaccins. La recherche a montré que le contenu négatif en matière de vaccins sur les médias sociaux entraîne une plus grande participation des utilisateurs que le contenu positif (16). Deuxièmement, nous n'avons pas examiné chaque commentaire pour identifier des comptes automatiques, plus précisément des "robots". Les robots sont définis comme des comptes automatisés qui peuvent être conçus pour diffuser de la désinformation et du contenu anti-vaccination (51). Yuan et al. ont découvert que $1,45 \%$ des comptes participant au discours sur les vaccins sur les médias sociaux étaient des robots (52). Troisièmement, seules les publications en anglais ont été incluses dans l'analyse et ne sont donc pas représentatives de la population non anglophone en général. Bien que les données aient été codées indépendamment par chacun des trois chercheurs afin de réduire le biais (53), nous n'avons utilisé que les publications sur les médias sociaux et nous n'avons donc pas pu trianguler les 
résultats de sources d'information multiples. Enfin, nous n'avons pas pu recueillir de renseignements démographiques auprès des commentateurs et, par conséquent, nous n'avons pas pu tirer de conclusions sur la généralisation des résultats à la population canadienne. Les recherches futures dans ce domaine devraient tenir compte de multiples méthodes de collecte de données pour vérifier la validité par l'analyse de l'information provenant de plusieurs sources, examiner le discours de médias sociaux dans des langues autres que l'anglais et sur d'autres plateformes de médias sociaux.

\section{Orientations futures}

Les résultats de cette étude peuvent aider à éclairer les messages sur les vaccins contre la COVID-19 de Santé Canada. Des recherches antérieures ont montré que les communications de santé publique peuvent avoir un effet positif sur l'intention de vaccination (22), et les thèmes dégagés dans cette étude concordent avec les recherches antérieures qui visaient à déterminer les messages efficaces sur les vaccins. En effet, il est impératif d'accroître les connaissances du public sur la gravité de la COVID-19 et l'innocuité des vaccins, étant donné qu'il s'agissait de préoccupations primordiales des auteurs de commentaires dans le cadre de cette étude et des participants à des recherches antérieures $(42,49,54,55)$. De plus, nos résultats sont conformes à ceux de la documentation publiée $(43,48,50)$ qui démontrent que les fournisseurs de soins de santé peuvent être efficaces pour diffuser des communications fiables sur les vaccins. Ensemble, des efforts fructueux peuvent être déployés pour améliorer les messages sur la vaccination sur les médias sociaux afin de réduire l'hésitation à l'égard de la vaccination.

Une nouvelle campagne d'information publique est nécessaire pour promouvoir l'urgence publique liée à la vaccination comme outil important pour lutter contre la COVID-19 et ses variants. Notre analyse fait ressortir des recommandations clés qui pourraient aider à accroître l'adoption des vaccins et à réduire I'hésitation. Ce comprend les éléments suivants : 1) des messages de la santé publique visant à mieux faire comprendre au public le contenu des vaccins contre la COVID-19; 2) la mise à profit de la confiance du public dans les professionnels de la santé pour assurer la liaison entre la santé publique et le public canadien afin de communiquer les avantages des vaccins contre la COVID-19 et ses variants; 3) des infographies claires publiées par la santé publique qui mettent en évidence les avantages des vaccins pour ceux qui l'ont reçu; et 4) la publication d'histoires poignantes et faciles à comprendre de membres de la communauté locale présentant des symptômes résiduels de la COVID-19 qui peuvent susciter un lien émotionnel.

\section{Conclusion}

Une analyse du discours sur les vaccins contre la COVID-19 sur les médias sociaux a permis de dégager quatre thèmes liés aux perceptions, aux attitudes, aux croyances et aux intentions à l'égard des vaccins contre la COVID-19. II s'agissait de thèmes négatifs (préoccupations au sujet de la nécessité, de I'innocuité et de l'efficacité des vaccins contre la COVID-19) et positifs (confiance dans les vaccins contre la COVID-19 comme solution sécuritaire). À la lumière de ces constatations, des recommandations précises visant à réduire l'hésitation à l'égard de la vaccination ont été élaborées.

\section{Déclaration des auteurs}

L. T. - Diriger le projet, la conceptualisation et la conception de l'étude, de la méthodologie, de la collecte des données, de I'analyse formelle et de l'interprétation des données, rédaction, révision et création de la version finale

D. D. - Conceptualisation et conception de l'étude, de la méthodologie, de la collecte des données, de l'analyse officielle et de l'interprétation des données, rédaction, révision et rédaction de la version finale

L. A. - Méthodologie, analyse officielle et interprétation des données, rédaction, révision et rédaction de la version finale

Tous les auteurs ont examiné et approuvé l'article final.

Le contenu de l'article et les points de vue qui y sont exprimés n'engagent que les auteurs et ne correspondent pas nécessairement à ceux du gouvernement du Canada.

\section{Intérêts concurrents}

Les auteurs ne déclarent aucun intérêt concurrent.

\section{Financement}

L. Tang bénéficie en partie d'un financement du Conseil de recherches en sciences humaines.

\section{Références}

1. Organisation mondiale de la Santé. Appellation de la maladie à coronavirus (COVID-19) et du virus qui la cause. Genève, Suisse : OMS; 2020 (accédé 2021-01-18). https://www.who. int/fr/emergencies/diseases/novel-coronavirus-2019/technicalguidance/naming-the-coronavirus-disease-(covid-2019)-andthe-virus-that-causes-it

2. Vogel L, Eggertson L. COVID-19: A timeline of Canada's first-wave response. CMAJ; 2020 (accédé 2021-08-26). https:// cmajnews.com/2020/06/12/coronavirus-1095847/

3. World Health Organization. WHO Coronavirus (COVID-19) Dashboard. Geneva, Switzerland: WHO; 2021 (accédé 2021-01-18). https://covid19.who.int/

4. Santé Canada. Mise à jour quotidienne sur l'épidémiologie de la COVID-19. Ottawa (ON) : SC; 2021. https://sante-infobase. canada.ca/covid-19/resume-epidemiologique-cas-covid-19. html

5. Andre FE, Booy R, Bock HL, Clemens J, Datta SK, John TJ, Lee BW, Lolekha S, Peltola H, Ruff TA, Santosham M, Schmitt HJ. Vaccination greatly reduces disease, disability, death and inequity worldwide. Bull World Health Organ 2008;86(2):1406. DOI PubMed 
6. Frederiksen LSF, Zhang Y, Foged C, Thakur A. The Long Road Toward COVID-19 Herd Immunity: Vaccine Platform Technologies and Mass Immunization Strategies. Front Immunol 2020;11:1817. DOI PubMed

7. Koirala A, Joo YJ, Khatami A, Chiu C, Britton PN. Vaccines for COVID-19: the current state of play. Paediatr Respir Rev 2020;35:43-9. DOI PubMed

8. Ministry of Health (Ontario). COVID-19 Vaccine Approval Process and Safety. Version 3.0 - March 11, 2021. Toronto, ON: MHLTC; 2021. https://www.health.gov.on.ca/en/pro/ programs/publichealth/coronavirus/docs/vaccine/COVID-19_ vaccine_approval_process_safety.pdf

9. Santé Canada. Vaccins approuvés contre la COVID-19. Ottawa (ON) : SC; 2021 (accédé 2021-08-28). https://www.canada. $\mathrm{ca} / \mathrm{fr} /$ sante-canada/services/medicaments-produits-sante/ covid19-industrie/medicaments-vaccins-traitements/vaccins. html

10. Lin C, Tu P, Beitsch LM. Confidence and Receptivity for COVID-19 Vaccines: A Rapid Systematic Review. Vaccines (Basel) 2020;9(1):16. DOI PubMed

11. McGregor S, Goldman RD. Determinants of parental vaccine hesitancy. Can Fam Physician 2021;67(5):339-41. DOI PubMed

12. Vallis $M$, Glazer S. Protecting individuals living with overweight and obesity: attitudes and concerns toward COVID-19 vaccination in Canada. Obesity (Silver Spring) 2021;29(7):1128-37. DOI PubMed

13. Organisation mondiale de la Santé. Dix ennemis que l'OMS devra affronter cette année. Genève, Suisse : OMS; 2019 (accédé 2021-01-18). https://www.who.int/fr/news-room/ spotlight/ten-threats-to-global-health-in-2019

14. MacDonald NE; SAGE Working Group on Vaccine Hesitancy. Vaccine hesitancy: Definition, scope and determinants. Vaccine 2015;33(34):4161-4. DOl PubMed

15. Dredze M, Broniatowski DA, Hilyard KM. Zika vaccine misconceptions: A social media analysis. Vaccine 2016;34(30):3441-2. DOI PubMed

16. Puri N, Coomes EA, Haghbayan H, Gunaratne K. Social media and vaccine hesitancy: new updates for the era of COVID-19 and globalized infectious diseases. Hum Vaccin Immunother 2020;16(11):2586-93. DOI PubMed

17. Ahmed N, Quinn SC, Hancock GR, Freimuth VS, Jamison A. Social media use and influenza vaccine uptake among White and African American adults. Vaccine 2018;36(49):7556-61. DOI PubMed

18. Statistique Canada. La majorité des Canadiens ont l'intention de se faire vacciner contre la COVID-19, septembre 2020. Ottawa, ON : StatCan 2020 (accédé 2021-01-18). https:// www150.statcan.gc.ca/n1/daily-quotidien/201217/dq201217cfra.htm

19. Angus Reid Institute. More Canadians willing to roll up their sleeves right away as national COVID-19 vaccine rollout begins. Angus Reid; 2020. https://angusreid.org/canadacovid-vaccine-december/
20. Organisation mondiale de la Santé. Immunité collective, confinement et COVID-19. Genève, Suisse : OMS; 2020 (accédé 2021-08-28). https://www.who.int/fr/emergencies/ diseases/novel-coronavirus-2019/question-and-answershub/q-a-detail/herd-immunity-lockdowns-and-covid19?gclid=CjwKCAiAv_KMBhAzEiwAs-rX1HsVC9B_pYOXNteV yRyGubiprvdH9t2bTMfhgloRg8PnTAfa_KqOAhoCMooQAvD_ BwE

21. Bond $L$, Nolan T. Making sense of perceptions of risk of diseases and vaccinations: a qualitative study combining models of health beliefs, decision-making and risk perception. BMC Public Health 2011;11(1):943-56.

DOI PubMed

22. Mercadante AR, Law AV. Will they, or Won't they? Examining patients' vaccine intention for flu and COVID-19 using the Health Belief Model. Res Social Adm Pharm 2021;17(9):1596605. DOI PubMed

23. Neubaum G, Krämer NC. Monitoring the Opinion of the Crowd: Psychological Mechanisms Underlying Public Opinion Perceptions on Social Media. Media Psychol 2017;20(3):50231. DOI

24. Chun JW, Lee MJ. When does individuals' willingness to speak out increase on social media? Perceived social support and perceived power/control. Comput Human Behav 2017;74:1209. DOI

25. Wu TY, Atkin DJ. To comment or not to comment: examining the influences of anonymity and social support on one's willingness to express in online news discussions. New Media Soc 2018;20(12):4512-32. DOI

26. Henrich $\mathrm{N}$, Holmes $\mathrm{B}$. What the public was saying about the $\mathrm{H} 1 \mathrm{~N} 1$ vaccine: perceptions and issues discussed in on-line comments during the $2009 \mathrm{H} 1 \mathrm{~N} 1$ pandemic. PLoS One 2011;6(4):e18479. DOI PubMed

27. Saleh SN, Lehmann CU, McDonald SA, Basit MA, Medford RJ. Understanding public perception of coronavirus disease 2019 (COVID-19) social distancing on Twitter. Infect Control Hosp Epidemiol 2021;42(2):131-8. DOI PubMed

28. Kirzinger A, Kearney A, Hamel L, Brodie M. KFF health tracking poll-early April 2020: The impact of coronavirus on life in America. Kaiser Family Foundation; 2020 (accédé 2021-08-26). https://www.kff.org/coronavirus-covid-19/report/ kff-health-tracking-poll-early-april-2020/

29. Epstein K. Just $14 \%$ of Americans support ending social distancing in order to reopen the economy, according to a new poll. Business Insider; 2020. https://www.businessinsider. $\mathrm{com} /$ poll-most-americans-support-coronavirus-socialdistancing-measures-2020-4

30. Williams L, Gallant AJ, Rasmussen S, Brown Nicholls LA, Cogan N, Deakin K, Young D, Flowers P. Towards intervention development to increase the uptake of COVID-19 vaccination among those at high risk: outlining evidence-based and theoretically informed future intervention content. $\mathrm{Br} \mathrm{J}$ Health Psychol 2020;25(4):1039-54. DOI PubMed 
31. Riad $A$, Abdulqader $H$, Morgado $M$, Domnori $S$, Košœík $M$, Mendes JJ, Klugar M, Kateeb E, IADS-Score. Global Prevalence and Drivers of Dental Students' COVID-19 Vaccine Hesitancy. Vaccines (Basel) 2021;9(6):566.

DOI PubMed

32. Coe AB, Elliott MH, Gatewood SB, Goode JV, Moczygemba LR. Perceptions and predictors of intention to receive the COVID-19 vaccine. Res Social Admin Pharm 2021;s1551-7411(21):000164-09. DOI PubMed

33. Griffith J, Marani H, Monkman H. COVID-19 Vaccine Hesitancy in Canada: Content Analysis of Tweets Using the Theoretical Domains Framework. J Med Internet Res 2021;23(4):e26874-e. DOI

34. Zhang S, Pian W, Ma F, Ni Z, Liu Y. Characterizing the COVID-19 Infodemic on Chinese Social Media: exploratory Study. JMIR Public Health Surveill 2021;7(2):e26090. DOI PubMed

35. Bonnevie E, Gallegos-Jeffrey A, Goldbarg J, Byrd B, Smyser $J$. Quantifying the rise of vaccine opposition on Twitter during the COVID-19 pandemic. J Commun Healthc 2021;14(1):12-9. $\mathrm{DOI}$

36. Guntuku SC, Buttenheim AM, Sherman G, Merchant RM. Twitter discourse reveals geographical and temporal variation in concerns about COVID-19 vaccines in the United States. Vaccine 2021;39(30):4034-8. DOl PubMed

37. Dubé E, Gagnon D, MacDonald N, Bocquier A, Peretti-Watel $P$, Verger $P$. Underlying factors impacting vaccine hesitancy in high income countries: a review of qualitative studies. Expert Rev Vaccines 2018;17(11):989-1004. DOI PubMed

38. Ahmed W, Vidal-Alaball J, Downing J, López Seguí F. COVID-19 and the 5G Conspiracy Theory: Social Network Analysis of Twitter Data. J Med Internet Res 2020;22(5):e19458. DOI PubMed

39. Kim H, Han JY, Seo Y. Effects of Facebook Comments on Attitude Toward Vaccines: The Roles of Perceived Distributions of Public Opinion and Perceived Vaccine Efficacy. J Health Commun 2020;25(2):159-69. DOI PubMed

40. Clarke V, Braun V. Thematic analysis. J Posit Psychol 2017;12(3):297-8. DOI

41. Bowen GA. Grounded Theory and Sensitizing Concepts. Int J Qual Methods 2006;5(3):12-23. DOI

42. Sweileh WM. Bibliometric analysis of global scientific literature on vaccine hesitancy in peer-reviewed journals (1990-2019). BMC Public Health 2020;20(1):1252.

DOI PubMed

43. Yaqub O, Castle-Clarke S, Sevdalis N, Chataway J. Attitudes to vaccination: a critical review. Soc Sci Med 2014;112:1-11. DOI PubMed
44. Carlsen B, Glenton $C$. The swine flu vaccine, public attitudes, and researcher interpretations: a systematic review of qualitative research. BMC Health Serv Res 2016:16:203. DOl PubMed

45. Karlsson LC, Soveri A, Lewandowsky S, Karlsson L, Karlsson H, Nolvi S, Karukivi M, Lindfelt M, Antfolk J. Fearing the disease or the vaccine: the case of COVID-19. Pers Individ Dif 2021;172:110590. DOI PubMed

46. Björkman I, Sanner MA. The Swedish $A(H 1 N 1)$ vaccination campaign--why did not all Swedes take the vaccination? Health Policy 2013;109(1):63-70. DOI PubMed

47. Kim H-Y, Bershteyn A, McGillen JB, Braithwaite RS. Under what circumstances could vaccination offset the harm from a more transmissible variant of SARS-COV-2 in NYC? Trade-offs regarding prioritization and speed of vaccination. medRxiv. 2021:2021.01.29.21250710. DOI

48. Jennings W, Stoker G, Willis H, Valgardsson V, Gaskell J, Devine D, McKay L, Mills MC. Lack of trust and social media echo chambers predict COVID-19 vaccine hesitancy. medRxiv. 2021:2021.01.26.21250246. DOI

49. Driedger SM, Maier R, Furgal C, Jardine C. Factors influencing H1N1 vaccine behavior among Manitoba Metis in Canada: a qualitative study. BMC Public Health 2015;15(1):128. DOI PubMed

50. Kowal SP, Jardine CG, Bubela TM. "If they tell me to get it, I'll get it. If they don't...": immunization decision-making processes of immigrant mothers. Can J Public Health 2015;106(4):e230-5. DOl PubMed

51. Broniatowski DA, Jamison AM, Qi S, AlKulaib L, Chen T, Benton A, Quinn SC, Dredze M. Weaponized Health Communication: Twitter Bots and Russian Trolls Amplify the Vaccine Debate. Am J Public Health 2018;108(10):1378-84. DOI PubMed

52. Yuan X, Schuchard RJ, Crooks AT. Examining Emergent Communities and Social Bots Within the Polarized Online Vaccination Debate in Twitter. Soc Media Soc. 2019;5(3):2056305119865465. DOI

53. Archibald MM. Investigator Triangulation: A Collaborative Strategy With Potential for Mixed Methods Research. J Mixed Methods Res 2015;10(3):228-50. DOI

54. Hidiroglu S, Ay P, Topuzoglu A, Kalafat C, Karavus M. Resistance to vaccination: the attitudes and practices of primary healthcare workers confronting the $\mathrm{H} 1 \mathrm{~N} 1$ pandemic. Vaccine 2010;28(51):8120-4. DOI PubMed

55. Nowak GJ, Sheedy K, Bursey K, Smith TM, Basket M. Promoting influenza vaccination: insights from a qualitative meta-analysis of 14 years of influenza-related communications research by U.S. Centers for Disease Control and Prevention (CDC). Vaccine 2015;33(24):2741-56. DOI PubMed 